\title{
Contralateral preoperative templating of lower limbs' mechanical angles is a reasonable option
}

\author{
Christophe Jacquet $^{1,2} \cdot$ Pierre Laumonerie $^{3,4} \cdot$ Sally LiArno $^{5} \cdot$ Ahmad Faizan $^{5} \cdot$ Akash Sharma $^{1,2} \cdot$ Louis Dagneaux $^{1,2}$. \\ Matthieu Ollivier ${ }^{1,2}$
}

\begin{abstract}
Purpose In cases where the femur or tibia exhibits abnormal mechanical angulation due to degenerative changes or fracture, the contralateral leg is often used to complete preoperative templating. The aim of this study was to determine the degree of asymmetry between knee joints in healthy individuals and to determine whether it is affected by differing demographic parameters.

Methods A CT scan-based modelling and analysis system was used to examine the lower limb of 233 patients (102 males, 131 women; mean age $61.2 \pm 15.2$ years, mean body mass index $24.9 \pm 4.4 \mathrm{~kg} / \mathrm{m}^{2}$ ) The hip-knee angle (HKA), lateral distal femoral angle (LDFA), medial proximal tibial angle (MPTA), posterior proximal tibial angle (ppta) and posterior distal femoral angle (PDFA) were then calculated for each patient. Results were then analysed to calculate femoral symmetry based on absolute differences (AD) and percentage asymmetry (\%AS) using a previously validated method.

Results Our results do not demonstrate any considerable asymmetry (percentage of asymmetry $>2 \%$ ) for all the anatomical parameters analysed: HKA (mean $\mathrm{AD}=1.5^{\circ}$; mean $\left.\mathrm{AS} \%=0.8, \mathrm{n} . \mathrm{s}\right)$, MPTA $\left(\mathrm{AD}=1.1^{\circ}\right.$; $\left.\mathrm{AS} \%=1.3, \mathrm{n} . \mathrm{s}\right), \mathrm{PPTA}\left(\mathrm{AD}=1.4^{\circ}\right.$; AS $\%=1.0, \mathrm{n} . \mathrm{s})$, LDFA (AD $=1.2 \mathrm{~mm}$; AS $\%=1.4, \mathrm{n} . \mathrm{s})$ and PDFA (AD $=0.9^{\circ}$; AS $\%=1.0$, n.s). Gender and ethnicity were not associated with significantly higher AD asymmetry. A significant correlation of AD asymmetry was observed between BMI and HKA, BMI and MPTA, and between patients' age and the MPTA.

Conclusion This data demonstrate that there is a non-statistically significant mechanical angle asymmetry between the two lower limbs. In cases where contralateral templating is used, such asymmetry will induce minimal (if any) clinical differences. Level of evidence IV.
\end{abstract}

Keywords Mechanical axis $\cdot$ Lower limb $\cdot$ Asymmetry $\cdot$ Contralateral templating

Matthieu Ollivier

ollivier.matthieu@yahoo.fr

1 Aix-Marseille Université, CNRS, ISM, UMR 7287, 13288 Marseille Cedex 09, France

2 Department of Orthopedics and Traumatology, Institute of Movement and Locomotion, St. Marguerite Hospital, 270 Boulevard Sainte Marguerite, BP 29, 13274 Marseille, France

3 Department of Orthopedics, Hôpital Pierre-Paul Riquet, 31000 Toulouse, France

4 Anatomy Laboratory, Faculty of Medicine, 31000 Toulouse, France

5 Stryker, Mahwah, NJ, USA

\section{Introduction}

Preoperative templating is often used prior to total knee arthroplasty (TKA) and knee osteotomies [3, 11, 17, 18, $22,24]$ to plan the required surgery and correction. In cases where the femur or tibia exhibits abnormal mechanical angulation due to degenerative changes or fracture; side-to-side comparison of human joint anatomy appears to be common practice in the evaluation of surgical methods $[2,6,14,16$, $20,25]$. However, this approach assumes asymmetry of a subject's knee geometry from one side to the other to be negligible [7]. Limited data are available on the morphometric side differences of human knee joint dimensions, and more importantly the published results seem to be contradictory. Therefore, the validity of anatomic side-to-side comparisons 
might be questioned for their use in surgical practice $[6,7$, 23].

The principal aim of this study was to evaluate the degree of asymmetry of the left and right knee joints with a particular emphasis placed on the mechanical parameters used in surgical preoperative planning. A secondary aim was to determine the effect of differing patient demographics on the degree of asymmetry present.

It was hypothesized that there will be no significant differences (as defined as $<2 \%$ ) in morphometric dimensions between the right and left knees. Hence, the results of this research protocol will support the concept of obtaining reference data from the contralateral uninjured knee in orthopaedic practice, regardless of patients' demographic and morphological specificity.

\section{Materials and methods}

A CT scan-based modelling and analysis system composed of scans of over 15,000 bone segments was used for this study (SOMA, Stryker, Mahwah, New Jersey [21]). Using this system, the pelvis, bilateral femora, bilateral tibiae and patellae of 233 normal patients (102 males and 131 females, mean age $61.2 \pm 15.4$ years, mean BMI $24.9 \pm 4.4 \mathrm{~kg} / \mathrm{m}^{2}$ ) representing a range of ethnicities (Caucasian, Asian and African American) were examined (Table 1). CT scan selection was done after radiographic inspection to rule out bone and joint anomalies, specimens with substantial arthritis, bone deformities or evidence of previous surgery. The demographic parameters evaluated in our analysis included age, gender, height, weight, body mass index (BMI) and ethnicity.

The non-weight-bearing hip-knee-ankle angle (nwHKA), lateral distal femoral angle (LDFA), medial proximal tibial angle (MPTA), posterior distal femoral angle (PDFA) and the global posterior plateau tibial angle (PPTA) were then calculated for each patient and each knee. All measurements were constructed using algorithm-calculated constructions on the corresponding bone which were then mapped to each chosen bone from the database. This resulted in reproducible and consistent constructs for each specimen [12]. Previous accuracy and reproducibility analysis estimated that this system allows automated measurement of upper femur anatomy with a margin of error $<2 \mathrm{~mm}$ and $<1^{\circ}$ [4].

The nwHKA axes were defined from the knee centre to the femoral head centre and ankle centre, respectively. The mechanical axes were then projected onto the coronal plane and the angle between both axes determined. The distal femoral axis was then created from the most distal points on the medial femoral condyle and lateral femoral condyle. The lateral angle between the distal femoral axis and the mechanical axis in the coronal plane was then determined as the LDFA. The proximal tibial plane was then created by fitting over 35 points on the medial and lateral tibial compartments. The intersection of the proximal tibial plane and the coronal plane defined the frontal tibial axis. The medial angle between the coronal tibial axis and the tibial mechanical axis in the coronal plane was then determined as the MPTA. The intersection of the proximal tibial plane and the sagittal plane defined the sagittal tibial axis. The posterior angle between the sagittal tibial axis and the tibial mechanical axis in the sagittal plane was determined as the PPTA. Lastly, the axis connecting the anterior and posterior points, where the femoral condyles meet the metaphysis, was defined as the sagittal femoral axis. The posterior angle between the sagittal femoral axis and the femoral mechanical axis in the sagittal plane was defined as the PDFA (Fig. 1).

Side-to-side absolute differences (AD) were obtained by subtracting the result of the smaller value (left or right) from the result of the larger. Percent absolute asymmetry was determined using the following documented method:

Table 1 Demographic and anatomic parameters evaluated in our global series (left and right anatomic measurements are pooled together)

\begin{tabular}{lcc}
\hline Demographic parameters & Mean \pm SD & Range (min-max) \\
\hline Age (year) & $61.2 \pm 15.2$ & $20-92$ \\
Height $(\mathrm{kg})$ & $166.4 \pm 8.7$ & $141-194$ \\
Weight $(\mathrm{cm})$ & $69.5 \pm 14.9$ & $44-128$ \\
BMI $\left(\mathrm{kg} \mathrm{m}^{-2}\right)$ & $24.9 \pm 4.4$ & $16.5-51.4$ \\
\hline Anatomic parameters & Mean \pm SD & Range (min-max) \\
\hline nwHKA $\left(^{\circ}\right)$ & $180 \pm 2.5$ & $169.9-187.1$ \\
LDFA $\left(^{\circ}\right)$ & $85.7 \pm 2.1$ & $79.7-90.9$ \\
MPTA $\left(^{\circ}\right)$ & $85.8 \pm 2.1$ & $77.7-91.4$ \\
PDFA $\left(^{\circ}\right)$ & $87.1 \pm 1.9$ & $81.8-93.1$ \\
PPTA $\left(^{\circ}\right)$ & $84.2 \pm 2.6$ & -2.7 to 14.2 \\
\hline
\end{tabular}

$B M I$ body mass index, $n w H K A$ non-weight-bearing hip-knee-ankle angle, LDFA lateral distal femoral angle, MPTA medial proximal tibial angle, $P D F A$ posterior distal femoral angle, PPTA posterior plateau tibial angle 


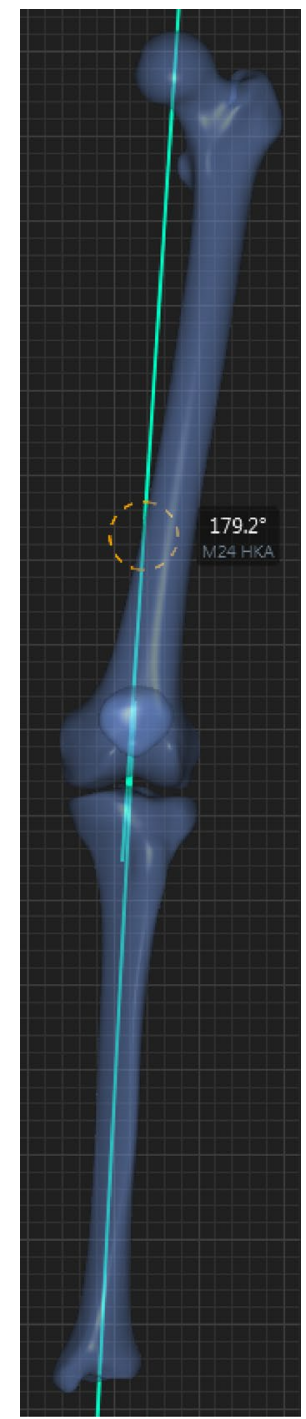

a

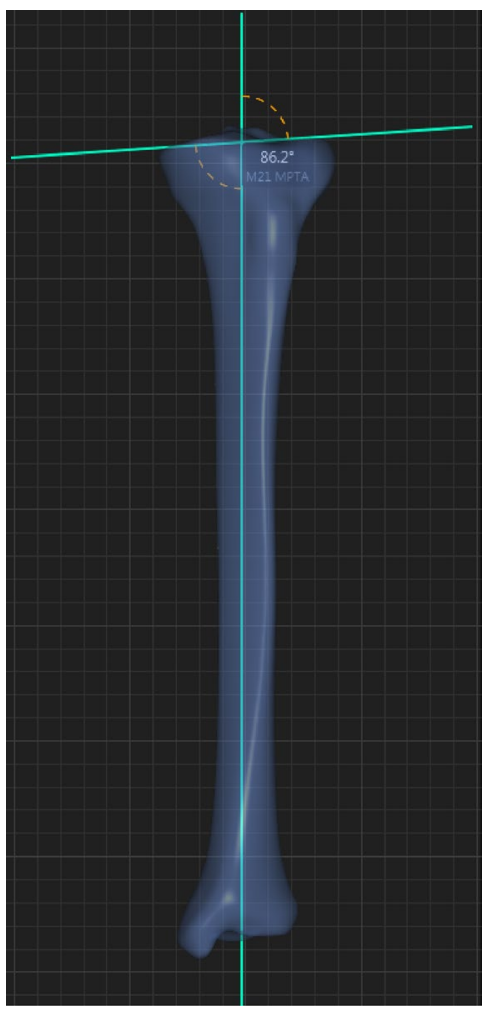

b

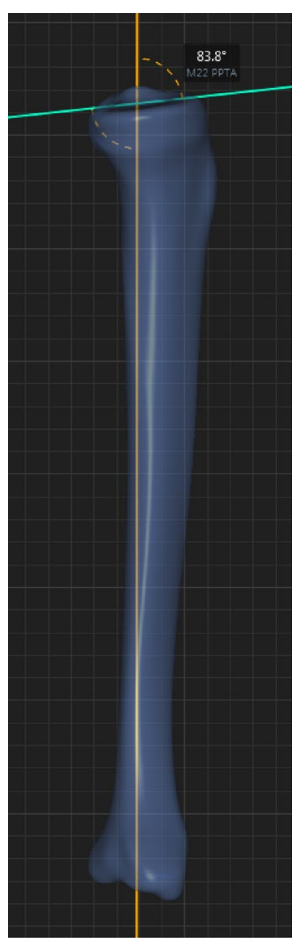

d

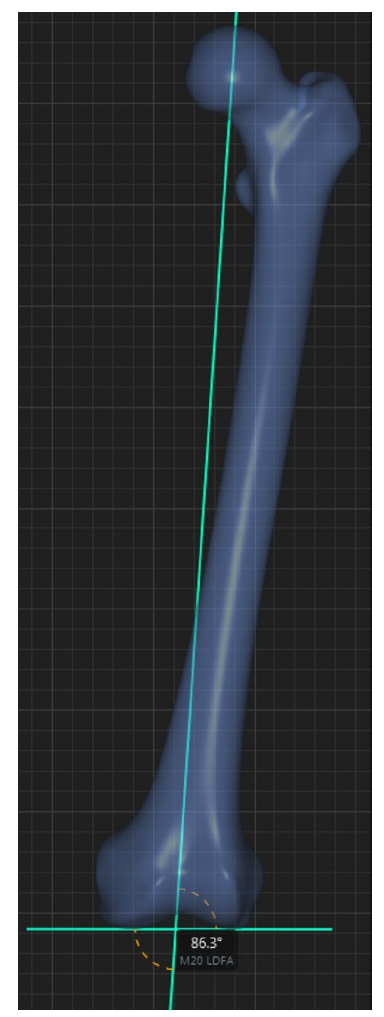

C

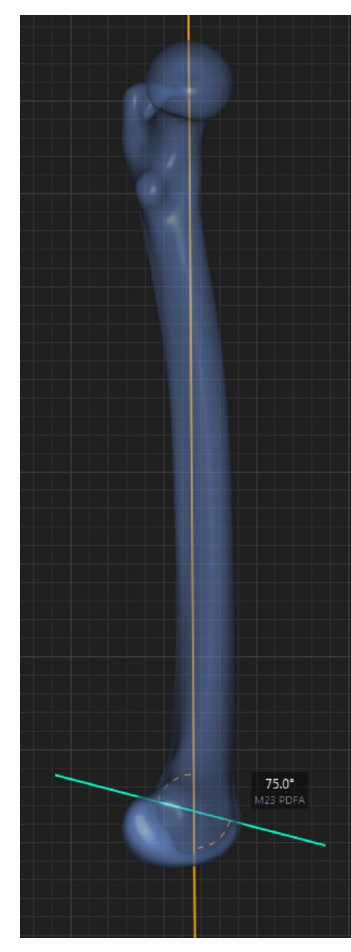

e

Fig. 1 Illustrations of our anatomical measurements. a $n w H K A$ non-weight-bearing hip-knee-ankle angle; b $M P T A$ medial proximal tibial angle; c $L D F A$ lateral distal femoral angle; d PPTA posterior plateau tibial angle; e $P D F A$ posterior distal femoral angle 
Percent asymmetry $=($ maximum $*$ minimum $) /($ average of maximum and minimum) [21]. This variable was used to define the magnitude of asymmetry, and we defined as "substantial asymmetry" side-to-side difference $>2 \%$ [12].

To perform this study, an anonymized database was explored following a local ethic committee approval of the research protocol.

\section{Statistical analysis}

Means and standard deviations were determined for each of the measurements made for the population as a whole and for various subpopulations, based on gender and ethnicity. Normal (Gaussian) distributions of the absolute differences and percent asymmetry between pairs of femora were determined. Univariate analysis was performed using $t$ tests. Pearson's coefficients were calculated to examine the correlations among absolute differences, specimen demographic data and femoral measurements. Multiple linear regression models were developed to establish the determinants for each of the variables that defined a difference between the left and right. For each model, variables with a $p$ value less than 0.1 were kept in the final model. Sample size was calculated based on the estimated MPTA measurement (MPTA $=87 \pm 3^{\circ}[25]$ ) for a required level of statistical significance of $\alpha=0.05$, and a power of $1-\beta=0.9 ; 48$ lower limb pairs would be required in each group or subgroups to detect $>2 \%$ asymmetry between knees. A trained statistician (MO) performed statistical analysis using SPSS software (Version 22; SPSS Inc, Chicago, IL, USA). All calculations were based on twotailed tests.

\section{Results}

Based on anatomical analysis of the 233 lower limb pairs, we found a mean nwHKA $180 \pm 2.5^{\circ}$, mean LDFA $85.7 \pm 2.1^{\circ}$, mean MPTA $85.8 \pm 2.1^{\circ}$, mean PDFA $87.1 \pm 1.9^{\circ}$ and mean PPTA $84.2 \pm 2.6^{\circ}$. No significant difference (all $p>0.05$ ) was found between the left and right knee anatomy if symmetry was estimated upon mean differences (Table 2) and a high degree of correlation (all $R^{2}>0.7$ ) was found between the left and right measurements for all parameters of interest (Table 3).

These results did not find any substantial asymmetry (percentage of asymmetry $>2 \%$ ) for all the anatomical parameters analysed: PPTA (mean $\mathrm{AD}=1.4 \pm 1.1^{\circ}$; mean As $\%=1 \pm 0.6 ; \mathrm{n} . \mathrm{s})$, PDFA ( $\mathrm{AD}=0.9 \pm 0.7^{\circ}$; As $\%=1.1 \pm 0.8$; n.s), LDFA ( $\mathrm{AD}=1.2 \pm 0.9^{\circ}$; As $\%=1.4 \pm 1.1$; n.s $)$, MPTA ( $\mathrm{AD}=1.1 \pm 0.9$; As $\%=1.3 \pm 1.1$; n.s), nwHKA $(\mathrm{AD}=1.5 \pm 1.2$; As $\%=0.8 \pm 0.7$; n.s) (Table 4).

Gender and ethnicity were not associated with a significantly higher $\mathrm{AD}$ asymmetry on univariate and multivariate
Table 2 Univariate analysis estimating difference of means left vs right

\begin{tabular}{llll}
\hline Anatomical parameters & $\begin{array}{l}\text { Difference of } \\
\text { means } \\
\text { (right vs left) }\end{array}$ & $\begin{array}{l}\text { 95\% Confi- } \\
\text { dence interval }\end{array}$ & $p$ value \\
\hline PPTA $\left(^{\circ}\right)$ & 1.0 & $0.9-1.1$ & n.s \\
PDFA $\left(^{\circ}\right)$ & 0.9 & $0.8-1.0$ & n.s \\
MPTA $\left(^{\circ}\right)$ & 1.1 & $1.0-1.2$ & n.s \\
LDFA $\left(^{\circ}\right)$ & 1.2 & $1.0-1.3$ & n.s \\
nwHKA $\left(^{\circ}\right)$ & 1.5 & $1.3-1.6$ & n.s \\
\hline
\end{tabular}

$n w H K A$ non-weight-bearing hip-knee-ankle angle, $L D F A$ lateral distal femoral angle, MPTA medial proximal tibial angle, $P D F A$ posterior distal femoral angle, $P P T A$ posterior plateau tibial angle, n.s not significant

Table 3 Degree of correlation between the left and right measurement for all parameters of interest

\begin{tabular}{llll}
\hline Variable by variable & $\begin{array}{l}\text { Degree of cor- } \\
\text { relation }\end{array}$ & $95 \%$ CI & $p$ value \\
\hline PPTA R vs PPTA L & 0.80 & {$[0.75-0.84]$} & $<0.01$ \\
PDFA R vs PDFA L & 0.85 & {$[0.81-0.88]$} & $<0.01$ \\
MPTA R vs MPTA L & 0.80 & {$[0.74-0.84]$} & $<0.01$ \\
LDFA R vs LDFA L & 0.74 & {$[0.67-0.79]$} & $<0.01$ \\
HKA R vs HKA L & 0.75 & {$[0.68-0.80]$} & $<0.01$ \\
\hline
\end{tabular}

$n w H K A$ non-weight-bearing hip-knee-ankle angle, $L D F A$ lateral distal femoral angle, MPTA medial proximal tibial angle, $P D F A$ posterior distal femoral angle, $P P T A$ posterior plateau tibial angle

analysis of all parameters (Table 5). We found a weak relationship between MPTA AD and age $(p=0.003)$ (Table 5). A significant correlation was also found between MPTA and nwHKA AD and BMI (respectively, $p=0.01$ and $p=0.02$ ). The same significant correlation was observed between MPTA and nwHKA AD and weight ( $p=0.01, p=0.03$ respectively), when height only correlated with MPTA AD $(p=0.04)$ (Table 5).

\section{Discussion}

The key finding of the current study is that there is no substantial asymmetry between the two lower limbs regarding the anatomical parameters analysed. These results support obtaining morphometric reference data from the contralateral uninjured side in the evaluation of unilateral knee pathologies.

Only few studies have evaluated side-to-side differences of anatomic structures of the knee joint in an attempt to support the use of side-to-side comparisons. Eckstein et al. [7] proposed that the articular surface area, the volume, as well as the local thickness of cartilage in human knee joints were 
Table 4 Calculation of side-to-side absolute difference (AD) and percentage of asymmetry $=($ maximum minimum $) /($ average of maximum and minimum) (\%As)

\begin{tabular}{lrcc}
\hline Anatomical parameters & Mean \pm SD & Range $(\min -\max )$ & $p$ value \\
\hline Left PPTA $\left(^{\circ}\right)$ & $83.9 \pm 2.9$ & $76-95.5$ & \\
AD & $1.4 \pm 1.1$ & $0.0-13.7$ & n.s \\
\%As & $1.0 \pm 0.6$ & $0.0-1.17$ & \\
Right PPTA $\left(^{\circ}\right)$ & $83.6 \pm 2.9$ & $75.3-92.7$ & \\
Left PDFA $\left(^{\circ}\right)$ & $87.4 \pm 1.9$ & $82.4-92.7$ & \\
AD & $0.9 \pm 0.7$ & $0-3.6$ & n.s \\
$\%$ As & $1.0 \pm 0.8$ & $0-4.2$ & \\
Right PDFA $\left(^{\circ}\right)$ & $87.2 \pm 2$ & $81.8-91.9$ & \\
Left LDFA $\left(^{\circ}\right)$ & $85.9 \pm 2$ & $80.1-92.5$ & \\
AD & $1.2 \pm 0.9$ & $0.0-4.4$ & n.s \\
\%As & $1.4 \pm 1.1$ & $0.0-5.4$ & \\
Right LDFA $\left(^{\circ}\right)$ & $85.5 \pm 1.9$ & $80.3-92.5$ & \\
Left MPTA $\left({ }^{\circ}\right)$ & $85.9 \pm 2.2$ & $77.5-98.4$ & \\
AD & $1.1 \pm 0.9$ & $0.0-14.5$ & n.s \\
\%As & $1.3 \pm 1.1$ & $0.0-17.3$ & \\
Right MPTA $\left({ }^{\circ}\right)$ & $85.9 \pm 2.2$ & $77.7-91.4$ & \\
Left nwHKA $\left({ }^{\circ}\right)$ & $179.9 \pm 2.6$ & $169.9-190$ & \\
AD & $1.5 \pm 1.2$ & $0.0-13.6$ & n.s \\
\%As & $0.8 \pm 0.7$ & $0.0-7.7$ & \\
Right nwHKA $\left(^{\circ}\right)$ & $180.2 \pm 2.7$ & $171.3-187.1$ & \\
\hline
\end{tabular}

"Substantial asymmetry" was defined as side-to-side difference $>2 \%$ (bold)

$n w H K A$ non-weight-bearing hip-knee-ankle angle, $L D F A$ lateral distal femoral angle, $M P T A$ medial proximal tibial angle, $P D F A$ posterior distal femoral angle, PPTA posterior plateau tibial angle, n.s not significant

Table 5 Results of our multivariate analysis regarding asymmetry influencing factors; all $p$ values calculated using multiple analysis of variance testing (MANOVA)

\begin{tabular}{llllll}
\hline $\begin{array}{l}\text { Confounding } \\
\text { factors for AD }\end{array}$ & PPTA & PDFA & LDFA & MPTA & nwHKA \\
\hline Gender & n.s & n.s & n.s & n.s & n.s \\
Age & n.s & n.s & n.s & $\begin{array}{l}p=0.003 \\
R^{2}=0.19\end{array}$ & n.s \\
BMI & n.s & n.s & n.s & $\begin{array}{l}p=0.01 \\
R^{2}=0.08\end{array}$ & $\begin{array}{l}p=0.02 \\
R^{2}=0.09\end{array}$ \\
& & & & $\begin{array}{l}p=0.04 \\
R^{2}=0.11\end{array}$ & n.s \\
Height & n.s & n.s & n.s & \\
Weight & n.s & n.s & n.s & $\begin{array}{l}p=01 \\
R^{2}=0.06\end{array}$ & $\begin{array}{l}p=0.03 \\
R^{2}=0.05\end{array}$ \\
Ethnicity & n.s & n.s & n.s & n.s & n.s \\
\hline
\end{tabular}

$R^{2}$ correlation coefficient, $A D$ absolute difference, $B M I$ body mass index, $n w H K A$ non-weight-bearing hip-knee-ankle angle, $L D F A$ lateral distal femoral angle, MPTA medial proximal tibial angle, $P D F A$ posterior distal femoral angle, PPTA posterior plateau tibial angle, $n . s$ not significant comparable between the right and left knees using magnetic resonance imaging. Based on their results, the authors supported obtaining reference data from the noninvolved side to estimate cartilage loss in unilateral osteoarthritis. In a recent study, Dargel et al. [6] studied 20 pairs of human cadaver knees. The coronal alignment, the morphometry of the articulating osseous structures of the femorotibial joint, the cruciate ligaments and the menisci were measured using established measurement methods and standard radiographs. This study has shown that for the femoral dimensions, there was a significant difference between the right and left knees in the anatomical lateral distal femoral angle (LDFA), with left knees $\left(80.3 \pm 2.2^{\circ}\right)$ displaying more valgus alignment than right knees $\left(82.1 \pm 2.4^{\circ}, p=0.022\right)$. Any other morphometric data on the femoral dimensions showed no significant differences. In the tibial dimensions, there was a significant correlation between the right and left knees in the width of the tibial plateau $(r=0.966, p \backslash 0.001)$ and the depth of the medial $(r=0.887, p<0.001)$ and lateral $(r=0.821, p<0.001)$ tibial condyles. But, their measurements were based only on plain radiographic evaluation of a small sample for the coronal limb alignment, and we feel that our study using CT scan of 233 patients would be more relevant to clinical practice $[3,17,18]$. In another cadaveric study, Schenk et al. [20] demonstrated small side differences in torsion between the left and right tibia, although these side differences in the coronal and sagittal plane were negligible. The present study confirms those conclusions and demonstrates no substantial side-to-side differences with regard to the set CT scan anatomical parameters used.

The hypothesis regarding the symmetry of lower limb's mechanical angles has important consequences in clinical practice. The satisfactory results in the short and medium term of kinematic alignment in prosthetic knee surgery have led to the promotion of individual correction based on the patient's morphological characteristics [1, 9, 10, 13]. The philosophy of implants' positioning in kinematic alignment surgery is aimed to restore the lower limb axis to its original anatomy prior to bony and articular deformation [10]. It requires complete $3 \mathrm{D}$ preoperative templating to obtain the different values of the tibial, femoral and lower limb's axis [19]. Thus, the use of the contralateral side may be necessary to restore this native alignment for peri-articular deformity associated with important bony or articular abnormalities such as malunion, wear or defects.

The same idea can be applied to lower limb axis correction during osteotomy surgery. Fürnstahl et al. [8] proposed the "intact" contralateral anatomy as a reconstruction landmark to estimate preoperative deformity correction in complex osteotomies following tibial plateau fracture. In their study, they demonstrated the accuracy of this technique by using the contralateral tibia as a $3 \mathrm{D}$ reconstruction template. In all of the three cases, they did not observe a significant 
residual error of correction by comparing the preoperative planning with the 3D model of the postoperative tibia. Victor et al. [26] described the use of 3D planning for axis correction of misaligned lower limbs with both varus and valgus deformities. If the contralateral leg had a normal morphology and alignment, it served as the template to be matched by the operated limb after the osteotomy. The mean deviation between the planned wedge angle and the executed wedge angle was $0^{\circ}$ ( -1 to 1, SD 0.72$)$ in the coronal plane and $0.3^{\circ}(-0.9$ to $3, \mathrm{SD} 1.14)$ in the sagittal plan. They proposed that the contralateral tibia seemed to be a reliable reconstruction template for the $3 \mathrm{D}$ preoperative planning of complex corrective osteotomies of the tibia.

Gender and the ethnicity were not associated with any absolute differences on univariate and multivariate analysis, confirming the use of contralateral templating as a valid option in male, female, Caucasian, Asian and African patients. With the number of knees available, we found asymmetry of MPTA and the nwHKA to positively correlate with BMI. We also found a significant correlation between the asymmetry of the MPTA and age. Older patients had more asymmetries regarding MPTA side-to-side measurement. However, these correlation values were very low and might only be statistically significant and not clinically relevant due to our statistical power in the entire cohort.

This study has several limitations. First due to the retrospective nature of our database evaluation, we were unable to evaluate information regarding patients' behaviour such as limb dominance and activity level, factors that could potentially correlate with asymmetry $[5,15]$. We only included subjects without bony abnormalities and our population does not represent the majority of patients requiring operative knee treatment. The purpose of this study was to establish the baseline side-to-side lower limb asymmetry of healthy subjects, as intact bony and cartilage morphology is mandatory for the proposed contralateral templating. On the other hand, although patients with bony deformities or disease, previous surgery or surgical hardware were excluded, patients with minor osteoarthritic, dysplastic or traumatic changes may have been included in our cohort. Finally, although the restoration of lower limb anatomy and function is one of the goals of TKA or osteotomy surgery, based on the variation observed, we cannot conclude on potential clinical consequence related to asymmetrical bias in TKA/ osteotomy templating.

Despite these limitations, this study used 3D modelling and analytical technology to automate the data process and achieve a highly accurate degree of morphometric analyses. This is further supported by the results of the validation step, where a deviation of generally less than $2 \mathrm{~mm}$ and $1^{\circ}$ was shown when compared with the mean of manually entered landmarks [4]. Another strength of this study is the number of pairs analysed (233), which is higher than the other studies analysing the side-to-side comparison of the knee geometry (20 in Dargel et al. [6], 15 in Eckstein et al. [7] and 51 in Schenk et al. [20]). Those numbers allowed us to perform secondary analyses (effect of demographic and morphometric parameters on asymmetry) with adequate statistical power.

The data from this study confirm that contralateral templating is a valid option in cases where the femur or tibia exhibits abnormal mechanical angulation due to degenerative changes or fracture.

\section{Conclusion}

These data demonstrate that there is a non-statistically significant mechanical angle asymmetry between the two lower limbs. In cases where contralateral templating is used, such asymmetry will induce minimal (if any) clinical differences.

Author contributions $\mathrm{MO}$ and PL designed the protocol. SL and FA made all CT measurements. MO and PL performed statistical analysis. $\mathrm{CJ}$ and LD wrote the initial draft. MO, LD and AS edited the different versions of the draft. CJ, PL, SL, FA, LD, MO and AS approved the submitted and final versions.

Funding No funding was needed for this study.

\section{Compliance with ethical standards}

Conflict of interest CJ,AS, LD and PL have nothing to disclose. SL and FA are employees of Stryker. MO is an educational consultant for Stryker.

Ethical approval The local ethics committee approved our study protocol prior to investigation.

\section{References}

1. Almaawi AM, Hutt JRB, Masse V, Lavigne M, Vendittoli P-A (2017) The impact of mechanical and restricted kinematic alignment on knee anatomy in total knee arthroplasty. J Arthroplasty 32:2133-2140

2. Anderson AF, Snyder RB, Federspiel CF, Lipscomb AB (1992) Instrumented evaluation of knee laxity: a comparison of five arthrometers. Am J Sports Med 20:135-140

3. Arnal-Burró J, Pérez-Mañanes R, Gallo-Del-Valle E, IgualadaBlazquez C, Cuervas-Mons M, Vaquero-Martín J (2017) Three dimensional-printed patient-specific cutting guides for femoral varization osteotomy: do it yourself. Knee 24:1359-1368

4. Banerjee S, Faizan A, Nevelos J, Kreuzer S, Burgkart R, Harwin SF, Mont MA (2014) Innovations in hip arthroplasty threedimensional modeling and analytical technology (SOMA). Surg Technol Int 24:288-294

5. Cooper C, Snow S, McAlindon TE, Kellingray S, Stuart B, Coggon D, Dieppe PA (2000) Risk factors for the incidence and 
progression of radiographic knee osteoarthritis. Arthritis Rheum 43:995-1000

6. Dargel J, Feiser J, Gotter M, Pennig D, Koebke J (2009) Side differences in the anatomy of human knee joints. Knee Surg Sports Traumatol Arthrosc 17:1368-1376

7. Eckstein F, Müller S, Faber SC, Englmeier K-H, Reiser M, Putz R (2002) Side differences of knee joint cartilage volume, thickness, and surface area, and correlation with lower limb dominance-an MRI-based study. Osteoarthr Cartilage 10:914-921

8. Fürnstahl P, Vlachopoulos L, Schweizer A, Fucentese SF, Koch PP (2015) Complex osteotomies of tibial plateau malunions using computer-assisted planning and patient-specific surgical guides. J Orthop Trauma 29:e270-e276

9. Hommel H, Abdel MP, Perka C (2017) Kinematic femoral alignment with gap balancing and patient-specific instrumentation in total knee arthroplasty: a randomized clinical trial. Eur J Orthop Surg Traumatol 27:683-688

10. Howell SM, Papadopoulos S, Kuznik KT, Hull ML (2013) Accurate alignment and high function after kinematically aligned TKA performed with generic instruments. Knee Surg Sports Traumatol Arthrosc 21:2271-2280

11. Jaffry Z, Masjedi M, Clarke S, Harris S, Karia M, Andrews B, Cobb J (2014) Unicompartmental knee arthroplasties: robot vs. patient specific instrumentation. Knee 21:428-434

12. Laumonerie P, Ollivier M, LiArno S, Faizan A, Cavaignac E, Argenson J-N (2018) Which factors influence proximal femoral asymmetry? Bone Joint J 100:839-844

13. Maderbacher G, Keshmiri A, Krieg B, Greimel F, Grifka J, Baier C (2018) Kinematic component alignment in total knee arthroplasty leads to better restoration of natural tibiofemoral kinematics compared to mechanic alignment. Knee Surg Sports Traumatol Arthrosc

14. Maderbacher G, Keshmiri A, Zeman F, Grifka J, Baier C (2015) Assessing joint line positions by means of the contralateral knee: a new approach for planning knee revision surgery? Knee Surg Sports Traumatol Arthrosc 23:3244-3250

15. Michaëlsson K, Byberg L, Ahlbom A, Melhus H, Farahmand BY (2011) Risk of severe knee and hip osteoarthritis in relation to level of physical exercise: a prospective cohort study of longdistance skiers in Sweden. PLoS One 6:e18339

16. Mochizuki T, Tanifuji O, Koga Y, Sato T, Kobayashi K, Nishino K, Watanabe S, Ariumi A, Fujii T, Yamagiwa H, Omori G, Endo
N (2017) Sex differences in femoral deformity determined using three-dimensional assessment for osteoarthritic knees. Knee Surg Sports Traumatol Arthrosc 25:468-476

17. Munier M, Donnez M, Ollivier M, Flecher X, Chabrand P, Argenson J-N, Parratte S (2017) Can three-dimensional patient-specific cutting guides be used to achieve optimal correction for high tibial osteotomy? Pilot study. Orthop Traumatol Surg Res 103:245-250

18. Pérez-Mañanes R, Burró JA, Manaute JR, Rodriguez FC, Martín JV (2016) 3D surgical printing cutting guides for open-wedge high tibial osteotomy: do it yourself. J Knee Surg 29:690-695

19. Roland M, Hull ML, Howell SM (2010) Virtual axis finder: a new method to determine the two kinematic axes of rotation for the tibio-femoral joint. J Biomech Eng 132:011009

20. Schenk P, Vlachopoulos L, Hingsammer A, Fucentese SF, Fürnstahl P (2018) Is the contralateral tibia a reliable template for reconstruction: a three-dimensional anatomy cadaveric study. Knee Surg Sports Traumatol Arthrosc 26:2324-2331

21. Schmidt W, LiArno S, Khlopas A, Petersik A, Mont MA (2018) Stryker orthopaedic modeling and analytics (SOMA): a review. Surg Technol Int 32:315-324

22. Stone AH, Sibia US, MacDonald JH (2018) Functional outcomes and accuracy of patient-specific instruments for total knee arthroplasty. Surg Innov 25:470-475 (1553350618787074)

23. Teitz CC, Lind BK, Sacks BM (1997) Symmetry of the femoral notch width index. Am J Sports Med 25:687-690

24. Thienpont E, Paternostre F, Pietsch M, Hafez M, Howell S (2013) Total knee arthroplasty with patient-specific instruments improves function and restores limb alignment in patients with extra-articular deformity. Knee 20:407-411

25. Thienpont E, Schwab PE, Cornu O, Bellemans J, Victor J (2017) Bone morphotypes of the varus and valgus knee. Arch Orthop Trauma Surg 137:393-400

26. Victor J, Premanathan A (2013) Virtual 3D planning and patient specific surgical guides for osteotomies around the knee: a feasibility and proof-of-concept study. Bone Jt J 95:153-158 